\title{
O ENSINO E O APRENDIZADO DAS HABILIDADES CLÍNICAS E COMPETÊNCIAS MÉDICAS
}

\author{
TEACHING AND LEARNING CLINICAL SKILLS AND MEDICAL COMPETENCES
}

\author{
Célia Maria Kira \& Milton de Arruda Martins
}

\begin{abstract}
Médica da Divisão de Clínica Médica do Hospital Universitário; Docente da Disciplina de Clínica Geral do Departamento de Clínica Médica da Faculdade de Medicina; Universidade de São Paulo

CORRESPONDÊNCIA: Prof.Dr. Milton de Arruda Martins - Faculdade de Medicina da Universidade de São Paulo - Rua Dr. Arnaldo, no 455, CEP: 01246-903 - São Paulo - SP
\end{abstract}

KIRA CM \& MARTINS M de A. O ensino e o aprendizado das habilidades clínicas e competências médicas. Medicina, Ribeirão Preto, 29: 407-413, out./dez. 1996.

RESUMO: O ensino médico de graduação deve visar o aprendizado não só de conhecimentos mas de habilidades e atitudes Para a formação de um profissional medico mais completo devem ser incorporadas ao ensino algumas tendências da Medicina Contemporânea, como o treinamento em ambulatório, a necessidade de levar em conta os custos nas decisões clínicas, a participação do paciente nas decisões que são tomadas a seu respeito, a abordagem multiprofissional do tratamento de saúde, o papel do médico na manutenção da saúde e prevenção das doenças, a importância dos conhecimentos de Epidemiologia à prática clínica e a valorização dos aspectos psicológicos, sociais e culturais, nas doenças e nos doentes.

Existem várias estratégias para o ensino do raciocínio clínico, sendo que uma combinação do reconhecimento de padrões e do raciocínio hipotético-dedutivo tem sido considerada mais adequada para o treinamento dos alunos de Medicina, para sua prática futura como médicos. Uma reflexão sobre essas estratégias tem levado a mudanças no curso de Semiologia Clínica da Faculdade de Medicina da Universidade de São Paulo, e elas são descritas neste artigo. Os autores comentam, ainda, a importância de algumas recomendações atuais, como o contato precoce dos estudantes com pacientes, o ensino em pequenos grupos, o aprendizado baseado em solução de problemas, o desenvolvimento da capacidade de buscar o conhecimento de forma autônoma, e a prática da Medicina baseada em evidências fornecidas pela investigação científica. Discutem também o papel do professor de Medicina que deve ser, ao mesmo tempo, médico competente, supervisor eficiente, provedor de informações, promotor do aprendizado e modelo adequado de atitudes e posturas, frente ao paciente e seus familiares e em relação a outros profissionais. cina.

UNITERMOS: Competência Clínica. Educação Médica. Educação de Pré-Graduação em Medi-

Uma vez que urna escola médica tem como finalidade principal à formação de médicos, o ensino ministrado durante os anos de graduação deve visar o aprendizado não apenas de conhecimentos, mas, de habilidades e atitudes. Trata-se de um processo complexo, mas que assume um papel central na formação de um bom médico. Este artigo contém algumas re- flexões sobre esse processo, em relação a como esse ensino deve ser estruturado e também a algumas tendências para o futuro.

A Medicina tem sido considerada como um conjunto de conhecimentos, habilidades e atitudes que a tomam ao mesmo tempo uma Ciência e urna Arte ${ }^{1,2}$. As bases científicas da Medicina devem fazer parte 
do ensino médico do primeiro ao último ano, de vendo ser sempre enfatizada a importância de uma prática de Medicina "baseada em evidências". Entretanto, existe um outro lado da prática médica, conhecido como a Arte da Medicina, que reúne intuição, reconhecimento das necessidades pessoais, culturais e sociais do paciente, compaixão, adequada relação médico-paciente, formação humanística, que devem também fazer parte integrante da formação do médico. $\mathrm{Na}$ verdade, esses dois aspectos da Medicina não devem ser considerados pelo estudante, separadamente, e ele deve aprender a ver o paciente como um todo, e a Medicina como a aplicação dos mais atuais conhecimentos científicos para o bem estar do paciente, de acordo com suas necessidades pessoais, culturais e sociais.

Ao se elaborar um currículo para uma escola médica, a definição inicial a ser feita é relativa ao tipo de médico que se deseja formar Não é uma definição fácil, mas os passos necessários à formação de um determinado produto só podem ser adequadamente definidos a partir da definição do produto a ser obtido. Essa definição não será necessariamente igual para todas as escolas médicas. Por exemplo, o objetivo de despertar, em uma parcela de alunos, o interesse pela carreira de investigação científica só pode ser adequadamente concretizado em escolas médicas que tenham uma tradição de pesquisa. A partir da definição mais geral do médico a ser formado, existe um segundo passo que será a definição das habilidades e competências mínimas que esse médico deve ter, para que o currículo seja pensado de forma a cumprir esses objetivos.

Algumas mudanças de ênfase têm sido introduzidas na prática médica, e devem, cada vez mais, ser incorporadas ao ensino médico ${ }^{4 / 7}$ :

- o treinamento em ambulatório e não apenas em enfermarias, serviços de emergência e unidades de terapia intensiva;

- a importância de, nas decisões clínicas, levar em conta os custos dos exames auxiliares, de internações hospitalares e de tratamentos propostos;

- a participação do paciente nas decisões que são tomadas a seu respeito;

- a importância de interação com uma equipe multiprofissional e não apenas com médicos;

- o papel do médico na manutenção da saúde, na prevenção primária e secundária e não apenas no diagnóstico e tratamento de doenças estabelecidas;
- a incorporação dos conhecimentos de Epidemiologia à prática clínica;

- o reconhecimento da importância dos aspectos psicológicos, sociais e culturais nas doenças e nos doentes.

\section{O ENSINO DE SEMIOLOGIA CLÍNICA}

Ao estabelecer um programa de ensino de técnicas de diagnóstico clínico, é importante analisar as estratégias que um médico usa para fazer o seu diagnóstico. Uma contribuição importante foi dada por Sackett e colaboradores, ao sistematizar definições e descrições de quatro estratégias possíveis para o diagnóstico clínico ${ }^{2}$.

1 - Reconhecimento de padrões - o médico usa sua experiência prévia e seus sentidos, principal mente visão, mas algumas vezes também audição ou mesmo olfato. Um dermatologista faz a maior parte de seus diagnósticos através do reconhecimento de padrões. Fazemos, às vezes, diagnóstico de doença de Graves, de anemia falciforme ou de depressão, ao observar um paciente entrando na sala. Muitas vezes, é difícil explicar a outros como foi feito um diagnóstico por reconhecimento de padrões. Um médico intensivista, às vezes, faz diagnóstico de septicemia, ao notar que o paciente está "toxemiado", mas tem dificuldade de descrever exatamente o que é "toxemia". O reconhecimento de padrões é o que, em outras épocas, era chamado de "olho clínico".

2 - Árvore de decisões - constitui-se esta estratégia diagnóstica em um conjunto de decisões que de vem ser tomadas, dependendo da resposta à urna questão anterior. Esta estratégia é também chamada de estratégia de múltiplas ramificações, ou "algoritmo" de decisões. Tem grande utilidade em algumas situações, por exemplo, triagem por nãomédicos ou protocolos de investigação, mas não é a forma através da qual os médicos habitualmente fazem o seu raciocínio clínico.

3 - História e o exame físico completos - esta é a for ma tradicional de ensinar a Semiologia Clínica. O aluno de Medicina, independente do que o paciente tem, faz uma história clínica a mais completa possível e o mesmo, com o exame físico, para depois tecer suas hipóteses diagnósticas e propor urna investigação laboratorial e uma conduta. Tem sido chamada também de estratégia da "exaustão". 
4 - Estratégia baseada no raciocínio hipotético-dedutivo. É a estratégia que os médicos adotam na sua atividade diária. Desde o momento em que o paciente entra na sala, o médico está formulando hipóteses diagnósticas e tentando confirmá-las ou excluí-las, pelo aspecto do paciente, pela sua for ma de falar, e, depois, através da história clínica e do exame físico. O raciocínio clínico não é, como muitas vezes é ensinado ao estudante de Medicina, um processo de duas fases, coleta de informações e reflexão sobre estas informações. É um processo muito mais dinâmico, em que, mesmo inconscientemente, o médico, desde o início, está formulando hipóteses.

Uma discussão sobre essas estratégias diagnósticas levou a uma modificação no ensino de Propedêutica Clínica na Faculdade de Medicina da Universidade de São Paulo. Passamos de um ensino em que o estudante devia fazer sempre uma história e um exame físicos completos (estratégia da "exaustão"), para um ensino em que o reconhecimento de padrões é estimulado e a história e os exames físicos são ensinados, levando-se em conta a estratégia do raciocínio hipotético-dedutivo.

Um aspecto a ser enfatizado, no ensino de Clínica Médica, é o papel relativo da história clínica, do exame físico e dos exames laboratoriais no diagnóstico e conduta. Existe uma impressão generalizada de que uma história clínica e um exame físico adequadamente realizados são capazes de levar o médico ao diagnóstico ou próximo dele, na grande maioria dos casos atendidos em ambulatórios ou consultórios. Entretanto, são escassos os estudos que tiveram como objetivo responder exatamente a esta questão. Em estudo realizado, na Inglaterra, por Hampton e colaboradores alguns médicos anotaram seu diagnóstico e possível conduta, após lerem a carta de encaminhamento, novamente após fazerem a história clínica e mais uma vez após realizarem o exame físico de pacientes em ambulatório. Esses diagnósticos e condutas propostas foram comparados com os diagnósticos e condutas adotadas dois meses após essa avaliação inicial. Um diagnóstico, feito após ler a carta de encaminhamento e realizar a história clínica, foi feito em 66 de 80 pacientes novos, $\mathrm{O}$ exame físico foi útil em mais sete pacientes e os exames de laboratório em apenas mais sete. Em apenas um em cada 6 pacientes em que o médico foi incapaz de fazer um diagnóstico após a história clínica e examinar o paciente, a investigação laboratorial levou a um diagnóstico positivo. Este estudo sugere que, na grande maioria do caso onde um diagnóstico correto é feito, este diagnóstico já está estabelecido (ou já era considerado alta mente provável), no momento em que a primeira consulta foi concluída. Em estudo similar, Sandler observou, em uma clínica de Medicina Geral, que, em 56\% dos pacientes, foi feito um diagnóstico correto ao final da história clínica e esta porcentagem subiu para $73 \%$, ao final do exame físico? .

Obviamente, à medida que se trata de pacientes mais graves, com múltiplos problemas ou doenças, exames laboratoriais tomam-se cada vez mais necessários e decisivos. Entretanto, mesmo nessas situações, a sua solicitação e interpretação deve ser baseada nos dados clínicos. Uma vez que o estudante de Medicina é exposto cada vez mais a uma enorme variedade de exames de laboratório e de imagem, e que presencia um comportamento, de muitos médicos, de valorização muitas vezes excessiva de alguns desses métodos, é importante que seja discutido o real papel da observação clínica no diagnóstico e acompanhamento dos pacientes. $O$ pensamento central talvez seja de que o reconhecimento de padrões, a história clínica e o exame físico, em conjunto, são a arma mais poderosa de que o médico dispõe para o diagnóstico e, muitas vezes, para o tratamento.

$\mathrm{Na}$ última década, foram relativamente poucos os estudos que tiveram corno objetivo testar, face aos novos recursos diagnósticos, sensibilidade, especificidade e valor preditivo de sinais e sintomas que têm sido usados classicamente, e que raramente passam por estudos desse tipo ${ }^{10,11,12}$. Na verdade, reconhecer o papel central da história clínica e do exame físico, na prática da Medicina Contemporânea, implica em investir recursos no sentido de estudar o seu exato papel. Uma implicação também direta dessa abordagem seria uma redução drástica dos crescentes custos da prática médica atual.

O curso de Semiologia Clínica da Faculdade de Medicina da Universidade de São Paulo, vem sendo continuamente modificado, no sentido de atingir a alguns dos objetivos descritos acima. Não existem aulas expositivas, sendo todo o curso feito em peque nos grupos. O curso é ministrado no quinto e sexto semestres, havendo um total de 60 períodos de aula. No semestre inicial, por vinte períodos (um por semana), cada grupo de alunos permanece com um único docente de Clínica Médica. Nos outros 10 períodos, existe um ensino mais dirigido de Semiologia Cardíaca, 
com docentes de Cardiologia. No segundo semestre, novamente os alunos permanecem por vinte períodos com um docente de Clínica Médica, e, por 10 períodos, com um docente de Neurologia, para um ensino mais dirigido à Semiologia Neurológica.

O ensino é feito sempre em contato com pacientes, sendo que, a partir de 1996, iniciou-se experiência de ensinar História Clínica, principalmente no ambulatório, e não mais na enfermaria, como tradicionalmente é feito. A enfermaria é um local talvez privilegiado para o ensino de técnicas de exame físico, reconhecimento de padrões, demonstração de situações em que o exame físico é alterado, e continua sendo usada com esse objetivo. Mas a história clínica exercitada a partir de pacientes de ambulatório, que apresentam problemas menos complexos, permite que o raciocínio hipotético-dedutivo possa ser exercitado pelos alunos, desde o início.

O ensino de Semiologia deve, em nosso entender, ser baseado em exposição contínua a pacientes e aprendizado a partir da discussão de como encaminhar a solução dos problemas que eles apresentam. É necessário, inicialmente, um treinamento nas técnicas de história clínica e de exame físico, mas, a partir dessa introdução, o aluno deve exercitar-se em fazer histórias clínicas, exame físico, estabelecer hipóteses diagnósticas e propor investigação laboratorial, caso seja necessário, sob supervisão de seu tutor.

\section{O CONTATO DOS ESTUDANTES COMPACIENTES}

Muito se tem discutido a respeito de qual seria o momento ideal para o estudante de Medicina iniciar o seu contato com pacientes. Existem dois aspectos a serem considerados: a motivação do estudante e a eficácia do aprendizado. A maioria das pessoas que iniciam um curso de Medicina tem por objetivo exercerem essa profissão no futuro, no atendimento direto a pessoas doentes. O estudante que inicia o curso médico vê a Medicina como uma prática do cuidado aos pacientes, e tem um grande interesse em estabelecer contato imediato com essa prática, tendo interesse menor nas bases científicas da Medicina. Muitos não compreendem a enorme importância dos conhecimentos básicos para a prática médica, $\mathrm{O}$ contato precoce com pacientes, neste contexto, seria uma motivação para o estudo e, nos cursos básicos, seria possível mostrar a relevância clínica dos conhecimentos de Biologia Molecular, de Anatomia e de Fisiologia. Por outro lado, haveria mais tempo para que as habilidades e atitudes necessárias para um relaciona- mento adequado com pacientes fossem amadurecendo. Em grande parte das escolas médicas do Brasil, o contato com pacientes é iniciado no quarto ou quinto semestres, mas há experiências bem sucedidas de início desse contato desde o primeiro semestre. No planejamento de novos currículos, vários autores defendem a idéia de que o contato dos alunos com pacientes seja o mais precoce possível.

\section{O ENSINO EM PEQUENOS GRUPOS}

Hoje não há dúvidas de que ensino de habilidades clínicas e competências médicas devem ser feitos em pequenos grupos, ficando os alunos sob a supervisão de um docente que permaneça com eles por um período prolongado. Assim como o ensino baseado em exposições teóricas a um número grande de alunos tem sido criticado, o ensino em pequenos grupos, em que, a cada sessão, os alunos entram em contato com um professor diferente, também apresenta sérios problemas, na medida em que não é estabelecido um vínculo entre o professor e o aluno, fundamental para que o processo de aprendizado tenha êxito ${ }^{3,6}$.

Em um ensino baseado em pequenos grupos (ensino tutorial), o professor desempenha um papel central e deve ser preparado para assumir este papel $^{14}$. Trata-se de um aspecto que tem sido pouco enfatizado nas escolas médicas. Além de competência na área do conhecimento que vai ensinar, o docente necessita de um preparo específico, para ser um bom tutor.

Segundo alguns autores, um professor de Medicina, dedicado ao ensino clínico, tem que desempenhar, ao mesmo tempo, quatro diferentes papéis: médico, devendo ter conhecimento e competência em sua área, e ter uma atitude apropriada em relação aos pacientes e seus familiares; supervisor, dando oportunidade aos estudantes de exercerem suas habilidades clínicas, enquanto os supervisiona; professor, trazendo novas informações, promovendo discussões e respondendo às dúvidas que vão surgindo; pessoa, estabelecendo um vínculo com os alunos que permita auxiliá-los no processo de aprendizado e nos problemas que vão surgindo nesse processo ${ }^{14}$.

O docente-médico exerce um papel que é funda mental, de modelo de atitudes e posturas em relação aos colegas, aos demais membros da equipe de saúde, ao paciente e a seus familiares. $\mathrm{Na}$ verdade, são esses docentes que vão ter uma influência decisiva nos comportamentos e atitudes que os estudantes de Medicina vão assumir, futuramente, como médi- 
cos. Por exemplo, para uma postura ética adequada, talvez sejam mais importantes às atitudes que os estudantes presenciam em seus professores na solução de problemas concretos que um curso formal de Ética Médica.

Tem sido demonstrado que o processo de aprendizado em Medicina é mais eficaz quando o estudante se defronta com problemas a serem resolvidos. Por outro lado, um ensino desse tipo tem sido considerado como mais adequado à formação de um médico, na medida em que, na prática diária, a atuação desse profissional é, principalmente, na solução de problemas apresentados por seus pacientes. Esse enfoque do ensino-aprendizado tem sido chamado de PBL ("problem-based learning"), sendo que o currículo médico de muitas escolas tem sido mudado no sentido de estruturar o ensino não mais, por disciplinas, mas, através da apresentação, aos alunos, de problemas cada vez mais complexos ${ }^{6}$.

O contato direto do estudante com o paciente, que é possível em países como o Brasil, deve ser intensamente aproveitado para o aprendizado clínico. Esse aprendizado pode ser totalmente estruturado a partir da discussão dos problemas que os estudantes vão encontrando em seu contato com pacientes.

Essa experiência contínua de relação estudante-paciente deve ser o núcleo no ensino clínico, mas pode ser complementada com equipamentos que permitem algumas simulações, que, em certos casos, são vantajosas. Dois exemplos que poderiam ser dados são sistemas de simulação de ausculta cardíaca, em que todos os sons cardíacos podem ser ouvidos várias vezes pelos estudantes, até que haja uma memorização completa de todas as suas características e diferenças; um outro exemplo é a existência de manequins para treinamento de exame pélvico feminino, sendo que os alunos podem treinar exaustivamente, sem o grande desconforto que isto poderia causar em pacientes.

\section{DESENVOLVIMENTO DA CAPACIDADE DE BUSCAR O CONHECIMENTO DE FORMA AUTÔNOMA}

Não há dúvida de que, hoje, um dos fenômenos mais importantes a influenciar a prática do médico é a explosão do conhecimento $\mathrm{O}$ conhecimento básico, de Bioquímica e Biologia Molecular, por exemplo, que é ministrado no primeiro ano do curso médico, estará, em parte, já ultrapassado, quando o aluno chegar ao sexto ano. A outra face deste fenômeno é que esse conhecimento, que cresce a uma velocidade cada vez maior, está, cada vez mais acessível, através de programas de ensino e atualização, bases de dados disponíveis através da Internet, programas para revisão bibliográfica através de computador. Seguramente, uma das competências mais importantes a serem adquiridas pelo estudante de Medicina é a capacidade de buscar o conhecimento de for ma autônoma, a capacidade de se atualizar continua mente, sabendo analisar criticamente a informação obtida. As escolas médicas estão ainda despreparadas para essa revolução da informação. Hoje é possível a um estudante de Medicina que, durante uma visita na enfermaria, teve urna dúvida e considerou que ela não foi solucionada pelo seu professor, fazer rapidamente, através de programas como o MedLine, um levantamento de todos os trabalhos publicados recentemente sobre o assunto, e, muitas vezes, estabelecer um diálogo, via Internet, com professores de outros centros médicos.

Essas são possibilidades que devem ser incentivadas, e, talvez, urna das mudanças mais importantes do ensino médico será a ênfase maior em dar ao aluno todos os instrumentos para que ele tenha autonomia, sistemática e crítica na busca do conhecimento. Urna das principais características de um bom médico é, e será cada vez mais, a sua capacidade de atualização constante, e formar médicos com essa habilidade deve ser um dos objetivos centrais do ensino médico, devendo as escolas médicas desenvolver estratégias específicas para esse treinamento

Nesse sentido, ainda são necessários estudos que possam definir com mais exatidão:

a- a melhor maneira de estruturar o aprendizado das habilidades de manusear adequadamente informação;

b- o impacto que essas habilidades podem ter nas decisões clínicas e em um melhor cuidado aos pacientes;

c- corno avaliar se essas habilidades foram adquiri das pelo estudante de Medicina.

\section{MEDICINA BASEADA EM EVIDÊNCIAS}

Nos últimos anos, têm surgido publicações relativas ao que alguns consideram uma nova abordagem do ensino e da prática da Medicina: a Medicina baseada em evidências (evidence-based medicine) ${ }^{18}$.

A experiência clínica e o desenvolvimento de "instintos" clínicos, em especial em relação ao diagnóstico, são uma parte importante e necessária da for- 
mação de um clínico competente. Muitos aspectos da prática clínica não podem ou não serão adequadamente testados. A experiência clínica é especialmente importante nessas situações. Por outro lado, estudos sistemáticos, que resultam em observações clínicas que podem ser reproduzidas, aumentam grandemente a confiança que um médico pode ter a respeito do prognóstico, valor de testes diagnósticos e eficácia de tratamentos. Antes de tomar uma decisão clínica, seria, então, importante verificar as evidências que existem a esse respeito, a partir de estudos previamente realizados. O estudante deveria então, sem desvalorizar a experiência clínica, ser treinado a buscar na Literatura resposta às suas dúvidas e aos problemas de seus pacientes.

O clínico competente deveria, então, regularmente consultar a Literatura e ser capaz de entender criticamente as seções de métodos e resultados dos trabalhos originais, e fazer com que esta busca constante de evidências o auxiliasse muito na solução de problemas clínicos e na tentativa de prestar cuidados de alto nível a seus pacientes.

Essa abordagem corresponde a uma valorização menor da autoridade. Um de seus pressupostos centrais é de que os médicos podem desenvolver habilidades de forma independente, obter os dados existentes na Literatura, e, desta forma, avaliar critica- mente as opiniões que são dadas por especialistas. Essa afirmação não implica em rejeição do que um estudante ou um médico pode aprender de professores e colegas mais experientes, cujos anos de experiência lhes deram habilidades únicas de obtenção de história clínica, exame físico e definição de estratégias para o diagnóstico. Muita dessa experiência jamais será obtida através do estudo de resultados de investigações científicas. Entretanto, essa abordagem incentiva o estudante a questionar legitimamente o conhecimento que lhe é transmitido.

Uma Medicina baseada em evidências leva a melhores decisões em relação a prognóstico e tratamento, por exemplo, mas as limitações de uma Medicina baseada unicamente em evidências são óbvias: a Medicina deve continuar a ser ensinada e praticada como uma tentativa de equilíbrio entre a Ciência e a Arte. Por outro lado, muitos dos pacientes reais que tratamos não seriam incluídos nos estudos em que nos baseamos para tratá-los: são muito velhos, são muito doentes, têm múltiplas doenças, apresentam efeitos colaterais dos medicamentos, apresentam dificuldades para seguir as recomendações, ou simplesmente não as aceitam. $\mathrm{O}$ estudante de Medicina deve continuamente aprender que cada paciente é um ser único, e, sempre, ser lembrado de que mais importante que a doença é o doente.

KIRA CM \& MARTINS M de A. Teaching and learning clinical skills and medical competences. Medicina, Ribeirão Preto, 29: 407-413, oct./dec. 1996

ABSTRACT: Undergraduate medical educations have to cover not only cognitive aspects but also skills and attitudes. In order to fulfill this objective, new trends have been incorporated into teaching, including training in outpatient clinics, notions of cost-efficiency analysis in clinical decision-making, multiprofessional team approach, the physicians role in health maintenance and prevention of disease, notions of clinical epidemiology and an increased value on psychosocial and cultural aspects related to diseases and patient's life. There are a number of ways to teach clinical reasoning, including a combination of thought and pattern recognition. These strategies have been taken into consideration to the introduction of changes in the teaching of basic clinical skills at the University of São Paulo Faculty of Medicine, which are briefly described in this paper. The authors also comment on the importance of some current recommendations, such as early student exposition to real patients, small group teaching, problem-based learning and self-learning skills and the practice of evidence-based Medicine. It is particularly stressed the role of medical teachers, who must be competent physicians, efficient supervisors, learning facilitators as well as suitable role models regarding positive attitudes towards the patient, his/her family and colleagues and other members of the health team.

UNITERMOS: Clinical Competence. Education, Medical. Education, Medical, Undergraduate. 


\section{REFERÊNCIAS BIBLIOGRÁFICAS}

1 - MENKEN M. The art of medicine revisited. Med Educ 28: 331332, 1994

2 - SACKETT DL et al. Clinical epidemiology: A basic science for clinical medicine. 2th ed. Little, Brown and Company, Boston, 1991.

3 - TOSTESON DC; ADELSTEIN SJ \& CARVER ST. New path ways to medical education. Learning to learn at Harvard Medical School. Harvard University Press, Cambridge, Massachusetts. 1994

4 - EICHNA LW. Medical school education for whom, student or patient. Bull N Y Acad Med 67:151-161, 1991.

5 - FRASER RC. Undergraduate medical education: present state and future needs. BMJ 303: 41-43, 1991.

6 - SCHMIDT HG et al. New directions for medical education. Problem-based learning and community-oriented medical education. Springer-Verlag, New York, 1989.

7 - STEARNS JA \& GLASSER M. How ambulatory care is different: a paradigm for teaching and practice. Med Educ 27: 3540, 1993.

8 - HAMPTON JR et al. Relative contributions of history-taking, physical examination, and laboratory investigation to diagnosis and management of medical outpatients. BMJ 2: 486-489, 1975.

9 - SANDLER G. The importance of the history in the medical clinics and the cost of unnecessary tests. Am Heart J 100: 928-931, 1980
10 - DEYO RA RAINVILLE J KENT DL. What can the history and physical examination tell us about low back pain? JAMA 268:760-765, 1992

11 - SACKETT DL. A primer on the precision and accuracy of the clinical examination. JAMA 267:2638-2644, 1992.

12- WILLIAMS JW \& SIMEL DL. Does this patient have ascites? How to divine fluid in the abdomen. JAMA 267:2645-2648, 1992.

13- CADE J. An evaluation of early patient contact for medical students. Med Educ 27: 205-210, 1993.

14 - ULLIAN JA; BLAND CJ \& SIMPSON DE. An alternative approach to defining the role of the clinical teacher. Acad Med 69: 832-838, 1994

15- JENNETT PA ei al. Preparing doctors for tomorrow: information management as a theme in undergraduate medical education. Med Educ 25:135-139, 1991.

16- CURRY RH \& MAKOUL G. An active-learning approach to basic clinical skills. Acad Med $71: 41-44,1996$.

17- DOMBAL FT. How do surgeons assimilate information? Theor Surg 1: 47-54, 1986

18- EVIDENCE-BASED WORKING GROUP. Evidence-based medicine: a new approach to teaching the practice of medicine. JAMA 268:2420-2425, 1992.

Recebido para publicação em 23/10/96

Aprovado para publicação em 11/12/96 